\title{
Vacuum-venipuncture skills: time required and importance of tube order
}

\author{
This article was published in the following Dove Press journal: \\ Vascular Health and Risk Management \\ 3 August 2013 \\ Number of times this article has been viewed
}

\section{Chieko Fujii \\ Faculty of Nursing and Medical Care, Keio University, Fujisawa, Japan}

Correspondence: Chieko Fujii Faculty of Nursing and Medical Care, Keio University, 44I I Endo, Fujisawa, Kanagawa 252-0883, Japan

$\mathrm{Tel}+8 \mathrm{I} 466496218$

Fax +8I 466496218

Email chieko@sfc.keio.ac.jp
Background: The purpose of this study was to assess specific vacuum-venipuncture skills and the influence of the time involved in skin puncture and blood collection.

Methods: Thirty subjects undergoing venipuncture in which video analysis was possible were included. These procedures were carried out by four nurses and recorded with a digital camera. Venipuncture skills classified by our observations were delineated on the basis of frame-byframe video images, and a graph of $x$ and $y$ coordinates was created.

Results: With the first blood-collection tube, strong blood flow required the practitioner to push the tube back in to compensate for the strong repulsive force in approximately $46 \%$ of cases. By the third blood-collection tube, the blood flow had weakened; therefore, the tube was moved up and down. In cases that required a second venipuncture, the tube was already pierced, so the time required to fill it to $5 \mathrm{~mL}$ was significantly longer.

Conclusion: Hand movement of the practitioner is adjusted according to blood flow. Reflex movement in response to strong blood flow may increase the risk of pushing the needle through the vein with excessive force. The time required to fill the tube varies among nurses, tube order, and level of venipuncture skills.

Keywords: blood collection, blood-collection tube, clinical practice, venipuncture skill

\section{Introduction}

Venipuncture provides important information for diagnosis and monitoring throughout the course of treatment. The sterility of stored whole blood depends on three factors: aseptic technique, a sterile system for collection and storage, and immediate, continuous refrigeration. ${ }^{1}$ It is likely that if the practitioner sampling the blood is not proficient, measurements will not be reliable.

Clinicians are occasionally confronted with the finding of an elevated serum or plasma potassium level in an otherwise healthy person because of the presence of occult mineralocorticoid deficiency or a defect in renal tubular transport. ${ }^{2}$ However, prolonged tourniquet time also results in a statistically significant elevation of serum bicarbonate, and underfilling of vacutainer tubes significantly influences the accuracy of serum bicarbonate values. ${ }^{3}$ Another consideration is that it is inappropriate to avoid using a tourniquet when performing venipuncture for calcium assays. ${ }^{4}$ When the volume of blood collected is less than required, accurate data may not be obtained because of a change in the ratio of drug to blood.

In vitro hemolysis depends mainly on how blood samples are drawn and treated, and it may depend specifically on the blood being forced through too fine a needle or through the large-bore needle of a syringe into a tube; it may also be caused by shaking 
the tube too vigorously and/or centrifuging the specimens before clotting is complete. ${ }^{5}$

With vacuum venipuncture, caution regarding flashbacks is warranted. Samples from the second bloodcollection tube are occasionally used for testing, because tissue fluid may contaminate the sample in the first tube. However, Raijmakers et $\mathrm{al}^{6}$ pointed out that the Clinical and Laboratory Standards Institute abandoned its recommendation for drawing a discard tube when performing a prothrombin time/international normalized ratio or an activated partial thromboplastin time, and that this can be extended to include more specialized plasma-based coagulation assays. Considering the contamination of blood-collection tubes by drugs, the order of tubes also becomes important.

Preanalytical activities, especially those directly connected with blood-sample collection and handling, are the most vulnerable steps throughout the testing process. ${ }^{7}$ These procedures must be analyzed more carefully to optimize sample accuracy. Although vacuum venipuncture is a basic skill, there are few experimental studies on venipuncture skills, particularly the time they require. This study aimed to clarify vacuum-venipuncture skills, as well as to identify those that influence the amount of time taken to perform this procedure.

\section{Materials and methods}

Of the yearly required health-checkup days scheduled for December 2011, three were scheduled for the study. If an individual was unwilling to cooperate on a particular day or if the time was inconvenient, another day was scheduled.

Approval for the study was obtained from the ethics committee of the Graduate School of Health Management, Keio University (June 23, 2010). Participants were provided with an explanation of the study objectives. All participants signed a written consent form.

\section{Subjects}

Four nurses who had worked in the hospital for longer than 10 years collected the blood samples from the subjects. The nurses were asked if the vacuum-venipuncture technique was their first choice, and they could select the best device for each subject, such as a syringe and a needle or a syringe and a butterfly needle. Subjects were asked to provide their age and sex.

Subjects chosen were those for whom the vacutainer tubes were used in the same order $(5 \mathrm{~mL}, 5 \mathrm{~mL}, 2 \mathrm{~mL})$. Thirty subjects undergoing venipuncture in which video analysis was possible were included.

\section{Venipuncture equipment}

Our equipment was manufactured by Terumo, Tokyo, Japan. Venoject II holders (XX-VP010HD01) were used for the vacuum-tube procedures with straight 21 -gauge needles (MN-HD2138MS). The first and second blood-collection tubes were used for biochemistry tests of blood with separating gel (NP-SP0725; Nipro, Tokyo, Japan). The necessary volume of blood was $5 \mathrm{~mL}$. The third blood-collection tube contained ethylenediaminetetraacetic acid- $2 \mathrm{~K}$, and the necessary volume of blood was $2 \mathrm{~mL}$ (NP-EK0205; Nipro). The tubes selected were the type with the conventional cover cap.

\section{Video recording}

A high-definition digital camera (HDR-HC9; Sony, Tokyo, Japan) mounted on a tripod and high-definition mini-DV tapes were used for recording the procedures. The maximum telephoto zoom $(10 \times)$ allowed a distance of $50 \mathrm{~cm}$ from the camera lens to the puncture point. This allowed optical image stabilization revision to avoid vibration. The puncture point was set directly under a pipe fluorescent lamp.

The procedure was captured using the video-editing software Edius Pro 5 (Grass Valley, Kobe, Japan), and the AVI files were transferred to a PC. To facilitate the acquisition process, we converted these files into 24-bit true color with a video capture of $720 \times 480$ pixels whilst matching them on a monitor. A sum-of-squared-differences correlationtracking algorithm was activated during acquisition to adapt the quality of the image sequence and account for differences in color.

\section{Video analysis}

Venipuncture skills were delineated on the basis of video images observed in every frame, and a graph of $x$ and $y$ coordinates was created by WINanalyze (Mikromak Service, Berlin, Germany), motion-analysis software that can track objects without markers. Time was determined by dividing the number of frames required by the frame rate of the AVI file (59.94 frames/second).

Figure 1 shows the time involved for each venipuncture technique and observation of the technique. Point A: the welded part of the needle and the needle base was a creamcolored point that was easy to recognize. Therefore, it was used to measure needle movement. Point B: measurement 

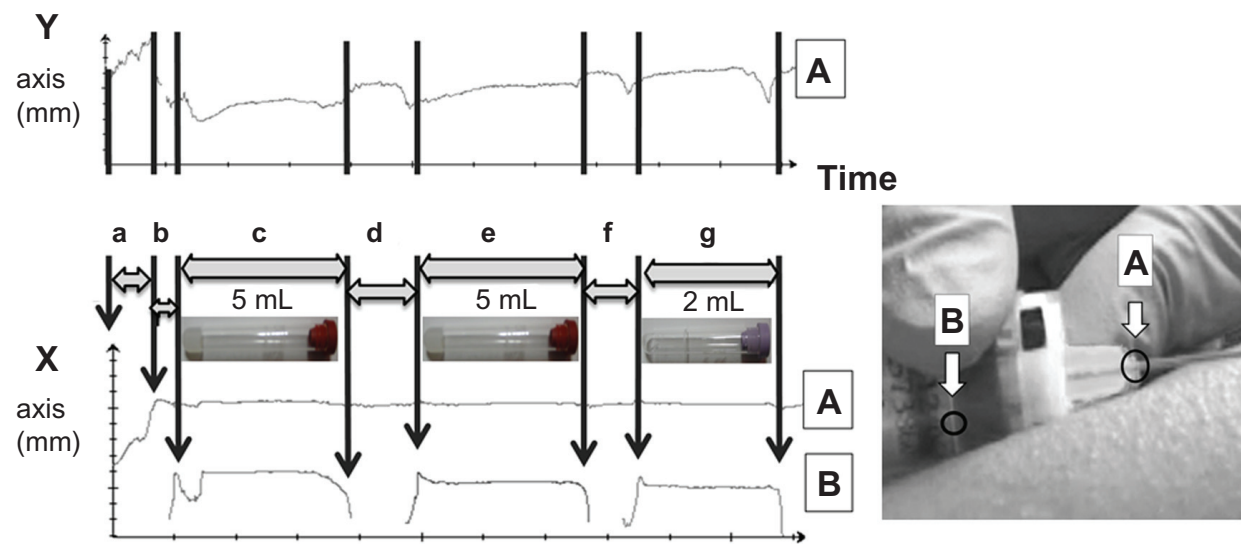

Time

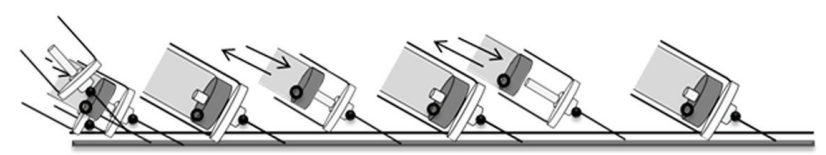

Figure I Using markerless measuring device. Venipuncture skills were delineated on the basis of video images observed frame by frame, and a graph of $x$ and $y$ coordinates was created by WINanalyze. Tracking points: A, the welded part of the needle and the needle base; B, the bottom of the rubber base of the tube. Venipuncture with time course: a, puncture; b, needle enters the vein; $c$ and e, first or second blood-collection tube $(5 \mathrm{~mL})$ with separating gel; $d$ and $f$, removed and inserted; $g$, the third bloodcollection tube $(2 \mathrm{~mL})$ contained ethylenediaminetetraacetic acid- $2 \mathrm{~K}$.

of movement of the tubes was performed from the bottom of the rubber base.

Delineation of the venipuncture steps and the time course:

a. puncture - from when the needle was inserted into the skin to when it entered the vein

b. needle entered the vein - from when the needle entered the vein to when the first tube was inserted into the holder; in some cases, hands changed at this step

c. first blood-collection tube $(5 \mathrm{~mL})$ - from when the tube was placed into the tip of the holder and blood was collected to when it was detached from the tip of the holder

d. and f. removed and inserted - the first blood-collection tube was removed, and the next one was inserted

e. the second blood-collection tube was placed into the tip of the holder, blood was collected, and the tube was detached from the tip of the holder

g. the third blood-collection tube was placed into the tip of the holder, blood was collected, and the tube was detached from the tip of the holder.

These steps were evaluated from points A and B on the graph created by the $x$ axis of WINanalyze.

\section{Statistical analysis}

Data obtained from the $x$ and $y$ coordinates were saved in Excel (Microsoft, Redmond, WA, USA). Statistical analysis was performed with SPSS 21.0 for Windows (IBM, Armonk, NY, USA).

The time required by each delineated venipuncture item was based on frame number and was compared for each observation item. The observation item at the time of the first, second, or third blood collection was examined with Fisher's exact test. A comparison of the time taken for different observation items was performed with Student's $t$-test in the event of equal variances or Welch's method if unequal variances were found. Probability values of $<0.05$ were considered significant.

\section{Results}

\section{Contributions}

Of these 30 subjects, 27 recorded their sex: twelve were male $(41.7 \%)$, and 15 were female $(50.0 \%)$. Mean age was 42.3 years (standard deviation 12.0 years), excluding the three subjects who did not record their sex.

Observation items and comparison of skills according to tube order:

- number of times - the first or second venipuncture from the same subject undergoing venipuncture.

- position of the tip - is the tube being held by the tip of the holder while blood is flowing into the tube (to determine whether to hold in the tip)?

- reinsertion - has the tube been placed into the holder again after being detached from the holder 
tip (to determine whether to reinsert the tube in the holder tip)?

- movement up and down - was the tube moved up and down while blood was flowing into it (to determine whether to move it up and down)? (Figure 2).

The skills of the nurses varied. Position of the tip and movement up and down of the second $(P=0.008,0.002)$ and third $(P<0.001)$ tubes were not the same among nurses.

Data from the WINanalyze graph were confirmed and classified. The time taken to insert the tube into the holder and hold the tube by the tip of the holder was significantly shorter with the third tube than with the second tube $(P=0.026)$. Reinsertion of the first tube occurred in $46.4 \%$ of cases. The rate of the tube moving up and down in the holder was significantly higher with the third tube than with the second tube $(P=0.003$, Table 1$)$.

\section{Comparison of time taken for different observation items}

Of the 30 subjects who underwent venipuncture, blood was collected on the first puncture from 28 (Table 2). One case was hidden by movement of the finger when the third tube was removed.
In two subjects, the needle was withdrawn and the skin punctured a second time. The time taken to enter the vein during the second blood draw was shorter than that during the first. The time (mean \pm standard deviation) required for blood collection with the first tube was $7.15 \pm 1.80$ seconds, whereas that with the other tubes was $13.04 \pm 0.63$ seconds $(P<0.001)$. The time taken to complete blood collection up to and including the third tube at first puncture was $25.93 \pm 3.55$ seconds, and the maximum time was 43.88 seconds. The time required for second puncture was longer than that for the first puncture (31.54 \pm 0.63 seconds, $P=0.038$ ).

With the second tube, holding it in at the tip $(6.73 \pm 1.66$ seconds) took less time than when the tube was not held in at the tip $(8.90 \pm 0.63$ seconds, $P<0.001)$, making the stick visible after the tube was inserted into the holder. Similarly, with the third tube, holding it in at the tip (5.97 \pm 2.49 seconds) took less time than when it was not held in at the tip $(8.13 \pm 2.27$ seconds, $P=0.028)$.

The time required to collect blood was $8.71 \pm 0.80$ seconds when the second tube was elevated and $6.86 \pm 1.72$ seconds $(P=0.029)$ when it was not. Similarly, the time required was $9.58 \pm 2.11$ seconds when the third tube was elevated and $6.59 \pm 2.30$ seconds when it was not $(P=0.009)$.

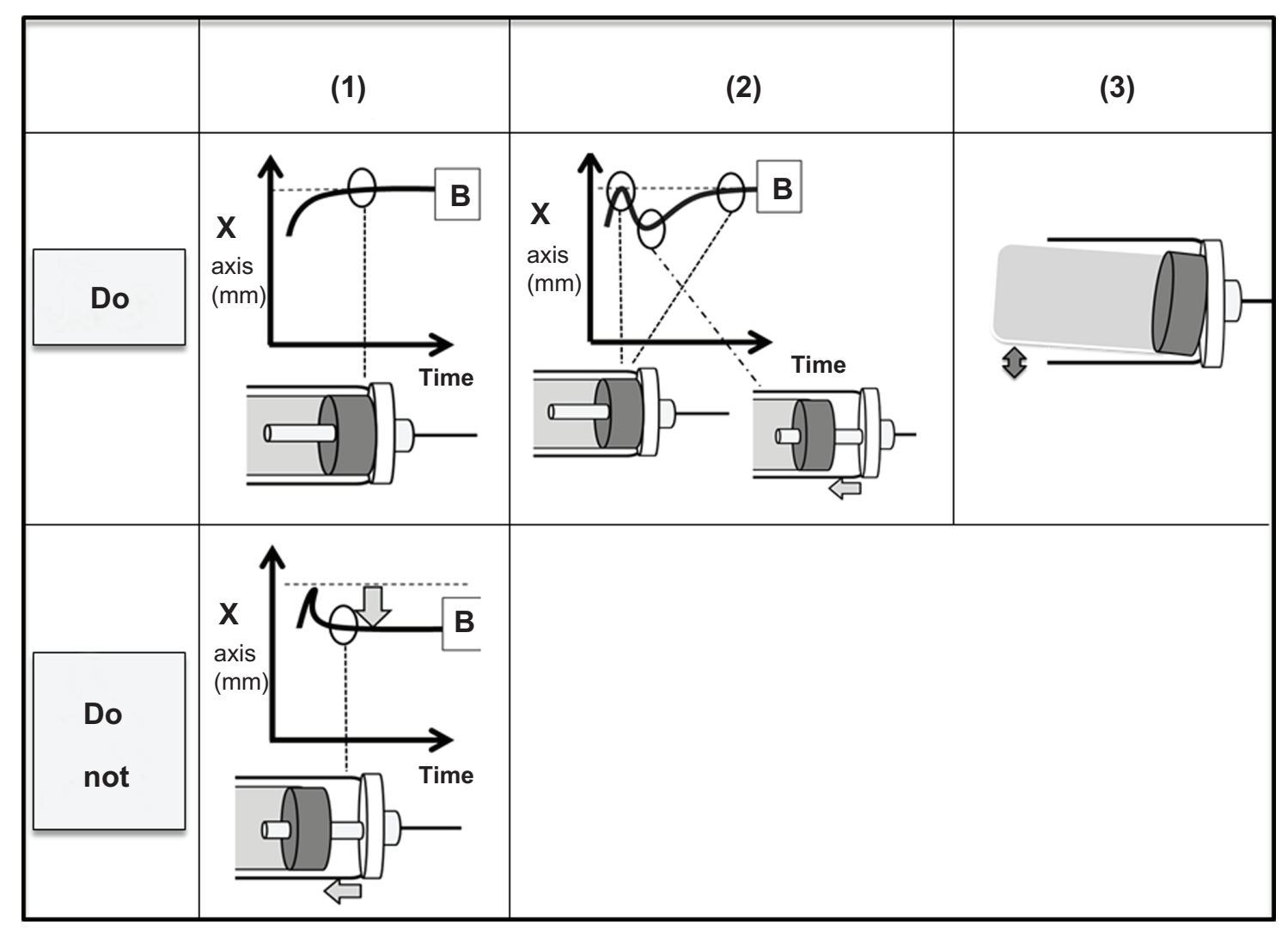

Figure 2 Observation items: position of the tip (I); reinsertion (2); movement up and down (3). Note: $B$ stands for the point at the bottom of the rubber base of the tube. 
Table I Comparison of techniques according to the order of blood collection tubes

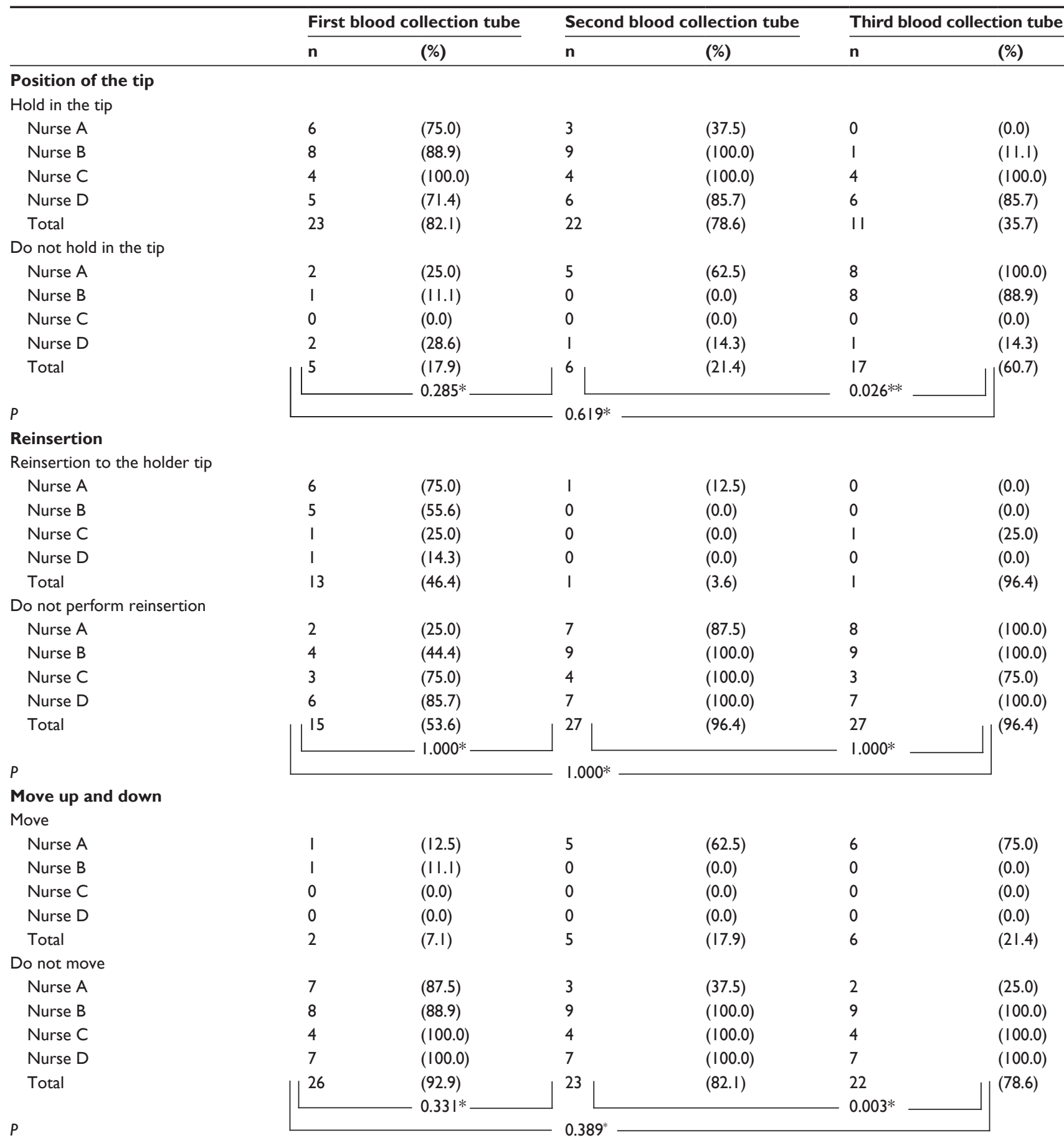

Note: *Fisher's exact test; **chi-square test; $n$, number of subjects.

\section{Effort involved in grasping the holder}

Pressure was applied to penetrate the rubber sleeve when inserting the blood-collection tube. Therefore, the motion of pushing the tube was performed with the dominant hand, and the distal interphalangeal joint of the nondominant index finger pushed the holder in the opposite direction (Figure 3).

\section{Discussion}

Advice on tourniquet time is included in phlebotomy training within the hospital, hence a campaign of appropriately channeled continuing education on this issue may be successful in reducing hemolysis rates. ${ }^{8}$ However, we were unable to find any studies documenting the time required to collect blood. Therefore, comparisons in relation to time could not 


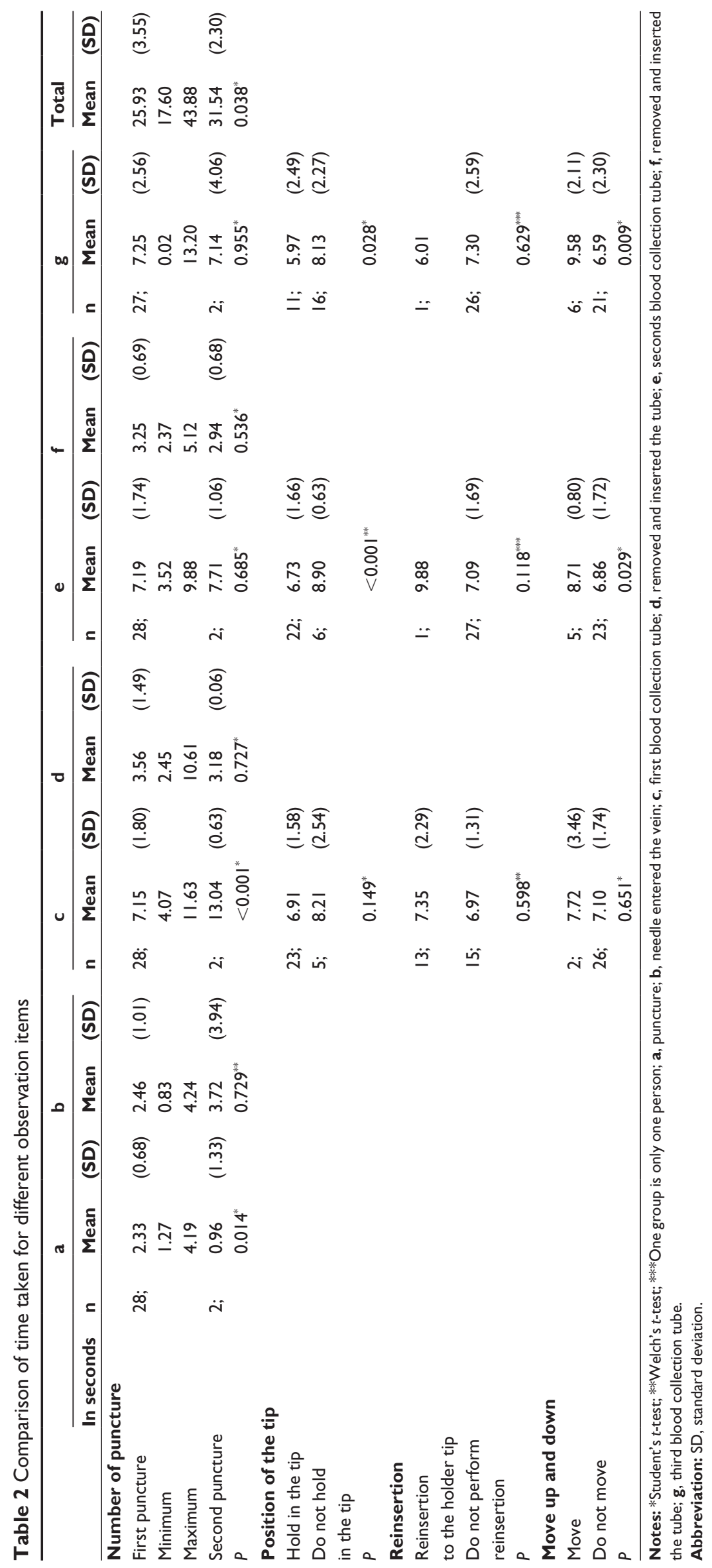




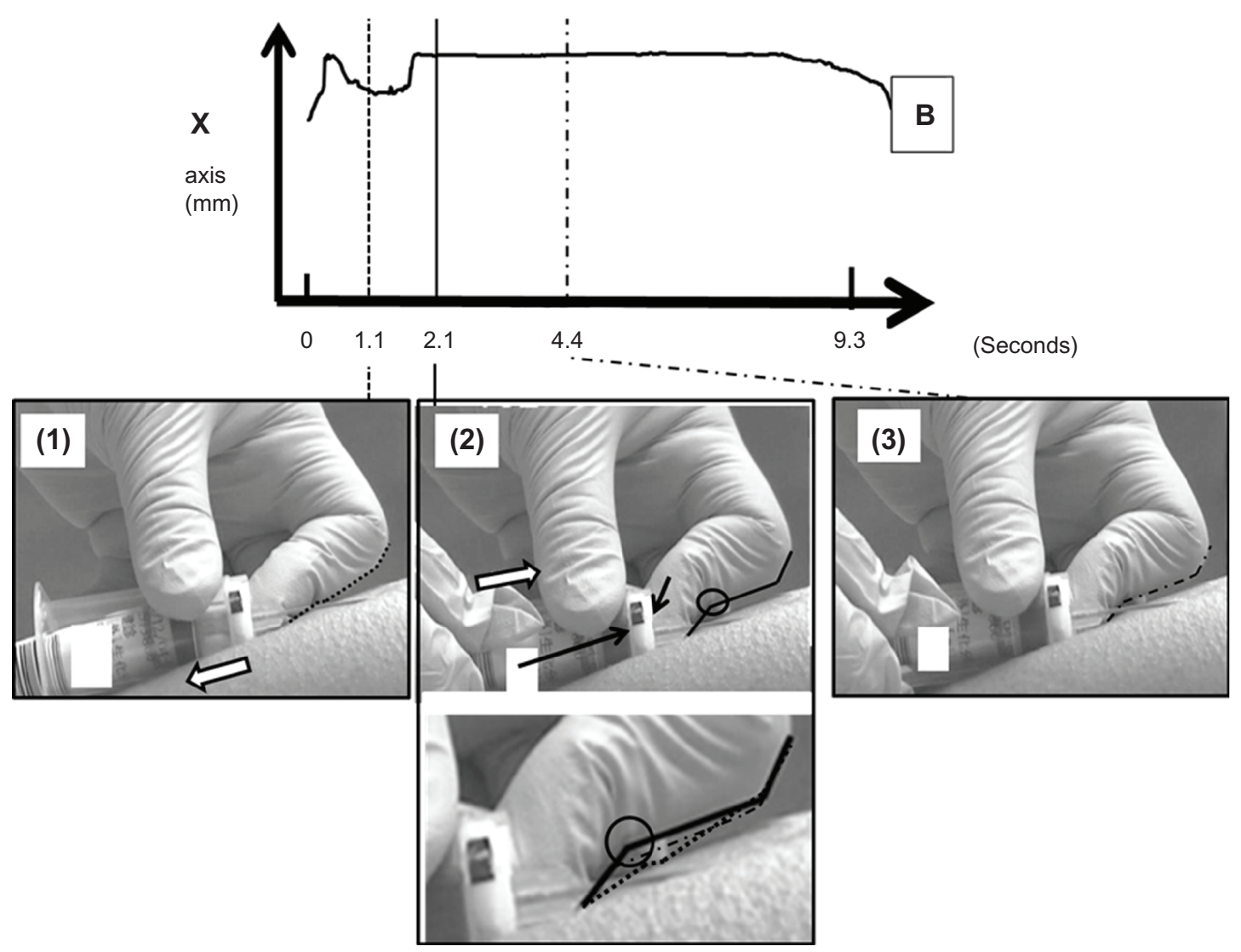

Figure 3 Characteristic movements used to grasp the holder: the first blood collection tube is detached (I); reinsertion of the blood-collection tube; the second finger of the left hand is pushed back so that the needle does not advance too much (2); the blood-collection tube is held with both hands (3).

Note: $B$ stands for the point at the bottom of the rubber base of the tube.

be performed. The average time required to complete blood collection up to and including the third tube was 26 seconds, and the maximum time was 44 seconds. In this study, it took 10 seconds or less to collect blood into one tube.

\section{Differences according to blood-collection order}

In this study, $5 \mathrm{~mL}$ tubes were used as the first and second tubes, and a $2 \mathrm{~mL}$ tube was used as the third. However, the time taken to fill each tube was roughly the same -7 seconds. The third tube included the time for tourniquet removal as well as movements to reach for sterilized cotton, which may have impacted the time. However, the third tube was moved up and down, and it was observed that these movements helped the inflow of blood, perhaps by weakening the backflow of blood.

Previous researchers have found that by adopting a singletube draw for coagulation testing, monetary savings can be realized with regard to purchasing equipment, disposal of extra draw tubes, and time. ${ }^{9}$ Care should be taken with the order of blood-collection tubes while performing venipuncture; filling the tubes with the required amount of blood is also important. Another basic training item is not to agitate the blood when dispensing it into the tubes. Furthermore, the coagulation test is usually carried out from the second tube onwards with the double-syringe method. Tissue fluid is believed to contaminate the first tube during vacuum venipuncture.

The rubber sleeve almost became detached, not only from insertion of the tube but also from the force of the backflow of blood. The nurses collecting the blood pushed the tube back in to compensate for this strong repulsive force for approximately $46 \%$ of the first tubes. This was observed to reflect the power from the backflow and pushing back the needle. Inexperienced nurses may be at risk of breaking through a vein.

Rather than a real skill, hand movement aided the flow of the blood. Figure 3 shows that the distal interphalangeal joint of the index finger of the nondominant hand reacted, and the insertion force and balance of the tube appeared to be maintained by the right hand. When force was required to push the tube in, the nurses applied pressure in the opposite direction so that the needle did not advance. 


\section{Collection of the appropriate volume into the blood-collection tube}

In two cases, blood did not flow into the tube after puncture. Therefore, the needle was removed and the nurse started again. The needle was exchanged for a new sterilized needle, and blood was collected. The second puncture was successful in both cases. However, because the tube had been pierced previously, the time taken to fill it to $5 \mathrm{~mL}$ was significantly longer because of the lack of negative pressure for the required volume. Alternatively, the obtained blood volume may have been insufficient. The technique to maintain the negative pressure required to draw the correct volume of blood should be clarified and learned as a basic skill.

\section{Standardization of the venipuncture procedure}

We could not carry out a comparison of test results in this study, and the impact of time was unclear. Moreover, the number of analyses was limited to 30 .

From these results, we must consider various aspects of venipuncture skills, such as determination of a fixed location for the tube in the holder among each nurse. We believe that the time taken for blood to enter the tube and the characteristics of blood over time contribute to the order of blood-collection tubes considered appropriate for testing purposes. Although venipuncture is a skill that is fostered and acquired by each individual, finding the best practice and standardizing it will further improve reliability.

\section{Disclosure}

The author reports no conflicts of interest in this work.

\section{References}

1. Borden CW, Hall WH. Fatal transfusion reactions from massive bacterial contamination of blood. N Engl J Med. 1951;245:760-765.

2. Don BR, Sebastian A, Cheitlin M, Christiansen M, Schambelan M. Pseudohyperkalemia caused by fist clenching during phlebotomy. N Engl J Med. 1990;322:1290-1292.

3. Berns SD, Matchett JL. Effect of phlebotomy technique on serum bicarbonate values. Acad Emerg Med. 1998;5:40-44.

4. McMullan AD, Burns J, Paterson CR. Venipuncture for calcium assays: should we still avoid the tourniquet? Postgrad Med J. 1990;66: 547-548.

5. Carraro P, Servidio G, Plebani M. Hemolyzed specimens: a reason for rejection or a clinical challenge? Clin Chem. 2000;46:306-307.

6. Raijmakers MT, Menting CH, Vader HL, van der Graaf F. Collection of blood specimens by venipuncture for plasma-based coagulation assays: necessity of a discard tube. Am J Clin Pathol. 2010;133:331-335.

7. Lippi G, Salvagno GL, Montagnana M, Lima-Oliveira G, Guidi GC, Favaloro EJ. Quality standards for sample collection in coagulation testing. Semin Thromb Hemost. 2012;38:565-575.

8. Saleem S, Mani V, Chadwick MA, Creanor S, Ayling RM. A prospective study of causes of haemolysis during venipuncture: tourniquet time should be kept to a minimum. Ann Clin Biochem. 2009;46:244-246.

9. McGlasson DL, More L, Best HA, Norris WL, Doe RH, Ray H. Drawing specimens for coagulation testing: is a second tube necessary? Clin Lab Sci. 1999;12:137-139.
Vascular Health and Risk Management

\section{Publish your work in this journal}

Vascular Health and Risk Management is an international, peerreviewed journal of therapeutics and risk management, focusing on concise rapid reporting of clinical studies on the processes involved in the maintenance of vascular health; the monitoring, prevention and treatment of vascular disease and its sequelae; and the involvement of

\section{Dovepress}

metabolic disorders, particularly diabetes. This journal is indexed on PubMed Central and MedLine. The manuscript management system is completely online and includes a very quick and fair peer-review system, which is all easy to use. Visit http://www.dovepress.com/ testimonials.php to read real quotes from published authors. 